\title{
Legionella RavZ Plays a Role in Preventing Ubiquitin Recruitment to Bacteria-Containing Vacuoles
}

\author{
Tomoko Kubori ${ }^{1,2 *}$, Xuan T. Bui ${ }^{1}$, Andree Hubber ${ }^{1}$ and Hiroki Nagai ${ }^{1,2 *}$ \\ ${ }^{1}$ Department of Infectious Disease Control, Research Institute for Microbial Diseases, Osaka University, Suita, Japan, \\ ${ }^{2}$ Department of Microbiology, Graduate School of Medicine, Gifu University, Gifu, Japan
}

\section{OPEN ACCESS}

Edited by:

Hayley J. Newton,

University of Melbourne, Australia

Reviewed by:

Hubert Hillbi,

University of Zurich, Switzerland

Thomas Naderer,

Monash University, Australia

Zhao-Qing Luo,

Purdue University, United States

*Correspondence:

Tomoko Kubori

tkubori@gifu-u.ac.jp

Hiroki Nagai

hnagai@gifu-u.ac.jp

Received: 06 June 2017 Accepted: 14 August 2017

Published: 28 August 2017

Citation:

Kubori T, Bui XT, Hubber $A$ and Nagai H (2017) Legionella RavZ Plays

a Role in Preventing Ubiquitin

Recruitment to Bacteria-Containing

Vacuoles.

Front. Cell. Infect. Microbiol. 7:384.

doi: 10.3389/fcimb.2017.00384
Bacterial pathogens like Salmonella and Legionella establish intracellular niches in host cells known as bacteria-containing vacuoles. In these vacuoles, bacteria can survive and replicate. Ubiquitin-dependent selective autophagy is a host defense mechanism to counteract infection by invading pathogens. The Legionella effector protein RavZ interferes with autophagy by irreversibly deconjugating LC3, an autophagy-related ubiquitin-like protein, from a phosphoglycolipid phosphatidylethanolamine. Using a co-infection system with Salmonella, we show here that Legionella RavZ interferes with ubiquitin recruitment to the Salmonella-containing vacuoles. The inhibitory activity is dependent on the same catalytic residue of RavZ that is involved in LC3 deconjugation. In semi-permeabilized cells infected with Salmonella, external addition of purified RavZ protein, but not of its catalytic mutant, induced removal of ubiquitin associated with Salmonella-containing vacuoles. The RavZ-mediated restriction of ubiquitin recruitment to Salmonella-containing vacuoles took place in the absence of the host system required for LC3 conjugation. These observations suggest the possibility that the targets of RavZ deconjugation activity include not only LC3, but also ubiquitin.

Keywords: Legionella, Salmonella, ubiquitin, vacuole, effector proteins, autophagy

\section{INTRODUCTION}

Legionella pneumophila is a Gram-negative bacterial pathogen that has a wide variety of eukaryotic hosts, ranging from amoebas to humans (Isberg et al., 2009). Host phagocytosis normally mediates entry of $L$. pneumophila into cells. Immediately after entry, L. pneumophila remodels the phagosome into a replicative niche, often referred to as a Legionella-containing vacuole (LCV). In order to establish the niche, L. pneumophila translocates $\sim 300$ effector proteins from bacteria into host cells via the specialized Dot/Icm type IV secretion system (T4SS), and by the coordinated function of these effector proteins it modulates a variety of host cellular processes (Ensminger and Isberg, 2009; Isberg et al., 2009; Hubber and Roy, 2010).

Similarly to other bacterial pathogens, L. pneumophila utilizes or modulates host ubiquitin pathways for its own benefit (Hubber et al., 2013; Zhou and Zhu, 2015). It was reported that L. pneumophila recruits ubiquitin to LCVs by a process depending on the Dot/Icm T4SS and bacterial protein synthesis (Dorer et al., 2006). The ubiquitin associated with LCVs is targeted for host proteasomal degradation, which was suggested to generate amino acids required for bacterial intracellular growth (Price et al., 2011). L. pneumophila possesses a large array of effector proteins involved in the modulation of the host ubiquitin system. 
Currently, several effector proteins have been found to possess the F-box and U-box domains implicated in E3 ubiquitin ligase activity (Kubori et al., 2008; Hubber et al., 2013). A recent structural analysis revealed that the bacterial protein SidC represents a novel family of E3 ubiquitin ligases (Hsu et al., 2014) containing a unique PI(4)P-binding domain (Luo et al., 2015). SidC associates with LCVs through its high affinity PI(4)Pbinding domain (Weber et al., 2006; Ragaz et al., 2008; Dolinsky et al., 2014) and has a direct or indirect role in ubiquitin recruitment to LCVs in early stages of infection (Horenkamp et al., 2014). L. pneumophila SidE family proteins exhibit not only deubiquitinase activity (Sheedlo et al., 2015) but also unusual ubiquitin ligase activity independent of the canonical E1 and E2 enzymes (Qiu et al., 2016; Kotewicz et al., 2017).

Autophagy is a conserved eukaryotic catabolic process for breakdown of intracellular components such as proteins or organelles. The target components are sequestered into a membrane-bound compartment called the autophagosome, which eventually fuses with a lysosome, a digestive compartment (Kuballa et al., 2012). Ubiquitin-like conjugation systems including Atg (Autophagy-related genes) proteins are crucially involved in autophagy (He and Klionsky, 2009). Microtubuleassociated protein light chain 3 (LC3), an Atg8 homolog in mammalian cells, is a well-known marker of autophagy that localizes in autophagosome membranes (Kabeya et al., 2000). The conjugation of LC3 to phosphatidylethanolamine (PE) on the early autophagosome structure is a critical step of autophagy (Ichimura et al., 2000).

Selective autophagy-mediated clearance of invading microbes is becoming recognized as a potent host immune response (Huang and Brumell, 2014). Salmonella enterica serovar Typhimurium has been well-studied as a model intracellular bacterium subjected to autophagy (Narayanan and Edelmann, 2014). Recognition of S. Typhimurium by the host autophagy machinery is thought to be mediated mainly by two signals: ubiquitin, which is associated with cytosolic bacteria or bacteriacontaining vacuoles (Birmingham et al., 2006), and a sugar $\beta$-galactoside on the luminal surface of bacteria-containing vacuoles that can be exposed by membrane rupture and is sensed by cytosolic receptor galectin-8 (Thurston et al., 2012). Recruitment of autophagic machinery to bacteria or bacterial vacuoles is mediated by LC3-binding adaptor proteins like p62/SQSTM1 and Nuclear dot protein $52 \mathrm{kDa}$ (NDP52) (Thurston et al., 2009; Deretic, 2010; Cemma et al., 2011). In addition to an LC3 binding site, p62 carries a ubiquitin binding site (Zheng et al., 2009), and NDP52 has both ubiquitin and galectin- 8 binding sites (Thurston et al., 2012). Another type of adaptor protein, Tecpr-1, which interacts with autophagy proteins Atg5 and WIPI2 (Atg18 homolog in mammalian cells), does not interact with ubiquitin or galectin-8 (Ogawa et al., 2011).

To counteract autophagy, bacteria have developed evasion strategies. The $S$. Typhimurium effector protein SseL deubiquitinates proteins on Salmonella-containing vacuoles (SCVs), preventing recognition by autophagic machinery (Mesquita et al., 2012). The L. pneumophila effector RavZ irreversibly deconjugates LC3 from PE (Choy et al., 2012), robustly inhibiting host autophagy. However, even in the absence of RavZ, L. pneumophila does not recruit LC3 on the vacuole (Hubber et al., 2017) and is highly resistant to clearance by selective autophagy, suggesting that $L$. pneumophila has alternative mechanisms for autophagy evasion (Choy et al., 2012; Rolando et al., 2016). The fact that L. pneumophila rarely recruits LC3 even in the absence of RavZ makes it difficult to identify alternative L. pneumophila factors inhibiting LC3 recruitment to LCVs. In an attempt to explore the possible alternative mechanisms, we used SCVs to examine potential roles of $L$. pneumophila effector proteins related to inhibition of selective autophagy. Using co-infection with L. pneumophila and $S$. Typhimurium, we unexpectedly found that RavZ plays a role in interfering with ubiquitin recruitment to the Salmonella-containing vacuoles.

\section{MATERIALS AND METHODS}

\section{Bacterial Strains, Plasmids, and Culture}

S. Typhimurium SR-11 x3181 carrying an mCherry-expressing plasmid (Hubber et al., 2014) was used for infection throughout the study. Legionella strains used in this study were derivatives of L. pneumophila strains Philadelphia-1 (Lp01) (Berger and Isberg, 1993). Salmonella was grown in Luria-Bertani (LB) medium containing $20 \mu \mathrm{g} / \mathrm{ml}$ of chloramphenicol to maintain the mCherry expressing plasmid. Legionella strains were grown in liquid N-(2-acetamido)-2-aminoethanesulfonic acid (ACES) buffered yeast extract (AYE) media (Horwitz and Silverstein, 1983). The rav $Z$ and $\operatorname{dot} A$ deletion strains were constructed by allelic exchange as described previously (Zuckman et al., 1999). Eukaryotic expression plasmids for RavZ were constructed by cloning the PCR-amplified wild-type or mutant (C258A) ravZ into the p3xFLAG-CMV-10 expression vector (Sigma). Sitedirected mutagenesis was conducted using the Quickchange II Site-Directed Mutagenesis Kit according to the manufacturer's recommendations (Agilent Technologies).

\section{Cell Cultures and Transfection}

HeLa-Fc $\gamma$ RII and HEK293T-Fc $\gamma$ RII cells were grown in Dulbecco's modified Eagle medium supplemented with 10\% fetal calf serum. Bone marrow derived macrophages (BMDMs) were prepared from A/J mice (Japan SLC, Inc.). The cells were plated onto cover glass in 24-well tissue culture plates 1 day before infection or transfection. Transfection was conducted using Lipofectamine 2000 (Invitrogen) or Fugene HD (Promega) according to the manufacturers' recommendations.

\section{Antibodies}

Mouse antibody against mono- and polyubiquitinylated conjugates (FK2) was purchased from Enzo (BML-PW8810). Mouse antibody against ubiquitin (P4D1) was purchased from Cell Signaling (\#3936). Mouse antibody against $\alpha$-tubulin was purchased from Sigma (T6074). Rabbit anti-Legionella antibody was purchased from Biodesign (B65051G). Secondary antibodies were purchased; Peroxidase-conjugated goat anti-mouse antibody (62-6520, Invitrogen), Alexa Fluor 488-conjugated goat anti-mouse antibody (A-11029, Thermo Fisher Scientific) 
and Rhodamine RedX-conjugated goat anti-rabbit antibody (R6394, Thermo Fisher Scientific).

\section{Bacterial Infections}

Overnight Salmonella culture was diluted 1:30 with fresh LB medium containing $20 \mu \mathrm{g} / \mathrm{ml}$ of Chloramphenicol, grown to an $\mathrm{OD}_{600}$ value of about 2.0, and used for infection. Overnight liquid culture of Legionella was used for infection. The bacterial cultures were spun down by microfuge, the culture media were replaced with phosphate-buffered saline (PBS), and the suspension was immediately used for infection. HeLa cells were infected with Salmonella with multiplicity of infection (MOI) 300 for $1 \mathrm{~h}$. After $20 \mathrm{~min}$ of infection, the cell culture medium was replaced with fresh medium containing $50 \mu \mathrm{g} / \mathrm{ml}$ of gentamycin to kill extracellular Salmonella. For the co-infection, a premixed solution of Salmonella and Legionella was prepared and immediately used for infection with MOI 300 of both bacteria for $1 \mathrm{~h}$. The time course study of Legionella infection in BMDMs was conducted with MOI 50. After $1 \mathrm{~h}$ of infection, the cells were washed three times with PBS to remove extracellular Legionella and the culture medium was replaced with fresh medium.

\section{Immunostaining}

Infected cells were fixed with $4 \%(\mathrm{w} / \mathrm{v})$ of paraformaldehyde (PFA) after three washes with PBS and permeabilized with chilled methanol or with $0.1 \%(\mathrm{w} / \mathrm{v})$ of Triton X-100 in PBS. After blocking with $2 \%(\mathrm{w} / \mathrm{v})$ of goat serum in PBS for $20 \mathrm{~min}$, the cells on the coverslips were reacted with a primary antibody for $1 \mathrm{~h}$, followed by a secondary antibody for $30 \mathrm{~min}$, at concentrations recommended by the manufacturers.

\section{Protein Purification}

BL21(DE3) carrying pET15b-ravZ (pNH1678) or pET15b$\operatorname{rav} Z_{\mathrm{C} 258 \mathrm{~A}}$ (pNH1679) was grown to logarithmic phase at $37^{\circ} \mathrm{C}$ in 11 of L-broth. After addition of IPTG to a final concentration of $0.4 \mathrm{mM}$ to induce the production of His-RavZ, cells were cultured overnight at $16^{\circ} \mathrm{C}$. E. coli cells were recovered by centrifugation and suspended in buffer A $(20 \mathrm{mM}$ Tris $\mathrm{HCl}$ pH 7.5, 5 mM EDTA) containing complete protease inhibitor cocktail (Roche). After treatment with $250 \mu \mathrm{g} / \mathrm{ml}$ lysozyme for 30 min, cells were disrupted by sonication. Lysate was centrifuged $(20,000 \mathrm{~g}, 20 \mathrm{~min})$ to remove insoluble materials and applied to Q-sepharose Fast Flow ( $\sim 25 \mathrm{ml}$ bed volume; GE Healthcase) equilibrated with buffer $\mathrm{B}(20 \mathrm{mM}$ Tris $\mathrm{HCl} \mathrm{pH} 7.5,10 \mathrm{mM} \beta$ mercaptoethanol) in an open column format. After washing with buffer B containing $200 \mathrm{mM} \mathrm{NaCl}$, the fraction containing HisRavZ was eluted with buffer B containing $300 \mathrm{mM} \mathrm{NaCl}$. This fraction was further applied to a HisSelect column $(1 \mathrm{ml}$ bed volume; Sigma-Aldridge) equilibrated with buffer C (20 mM Tris $\mathrm{HCl} \mathrm{pH} \mathrm{7.5,} 300 \mathrm{mM} \mathrm{NaCl}, 10 \mathrm{mM} \beta$-mercaptoethanol). After washing with buffer $\mathrm{C}$ containing $10 \mathrm{mM}$ imidazole, fractions were eluted with a linear gradient of $10-100 \mathrm{mM}$ imidazole in buffer C. Fractions containing His-RavZ were pooled and concentrated and subjected to a size exclusion column (Superdex 200 16/60 pg; GE healthcare). His-RavZ was eluted in 20 mM Tris $\mathrm{HCl} \mathrm{pH}$ 7.5, $300 \mathrm{mM} \mathrm{NaCl}, 1 \mathrm{mM}$ DTT.

\section{Semi-permeabilized Cells Experiment}

After $1 \mathrm{~h}$ of infection with Salmonella, HeLa cells were washed twice with semi-permeabilization buffer [125 mM K(OAc), $2.5 \mathrm{mM} \mathrm{Mg}(\mathrm{OAc})_{2}, 25 \mathrm{mM}$ Hepes-KOH pH 7.4, $1 \mathrm{mg} / \mathrm{ml}$ Glucose, $1 \mathrm{mM}$ DTT]. The cells were treated with $30 \mu \mathrm{g} / \mathrm{ml}$ digitonin in the semi-permeabilization buffer for $4 \mathrm{~min}$ at room temperature and washed another three times with the buffer. The permeabilized cells were treated with purified His-tagged wildtype or mutant RavZ proteins with gentle agitation for $1 \mathrm{~h}$ at room temperature. After two washes, the cells were fixed with $4 \%$ $(\mathrm{w} / \mathrm{v})$ PFA and subjected to immunostaining.

\section{Statistical Analysis}

Immunofluorescence experiments were conducted with at least 100 bacterial vacuoles counted per single experiment. Values were compared using paired Student's $t$-tests on three independent experiments.

\section{Ethics Statement}

All animal experiments were performed in accordance with the institutional guidelines and were approved by the Animal Care and Use Committee of the Research Institute for Microbial Diseases, Osaka University, Japan (Biken-AP-H26-10-0).

\section{RESULTS}

\section{LC3 Recruitment to Salmonella-Containing Vacuoles Is Inhibited by Co-infecting L. pneumophila}

As previously described, Legionella RavZ is sufficient to inhibit macroautophagy in mammalian cells, but it is not the only determinant preventing LC3 recruitment to LCVs (Choy et al., 2012). In an attempt to identify L. pneumophila protein factors responsible for the prevention of LC3 recruitment, we used an S. Typhimurium and L. pneumophila co-infection system. Selective autophagy targets a fraction ( 25\%) of Salmonellacontaining vacuoles (SCVs) at $1 \mathrm{~h}$ post-infection, as evidenced by the LC3-positive SCVs observed in GFP-LC3 expressing HeLa cells challenged with $S$. Typhimurium (Figure 1 SR-11). When HeLa cells were co-infected with wild-type L. pneumophila, LC3 recruitment to SCVs was reduced (Figure 1 SR-11+Lp01). In contrast, in HeLa cells co-infected with L. pneumophila strains lacking functional Dot/Icm T4SS or RavZ, LC3 recruitment to SCVs was not affected (Figure 1 SR-11+Lp01 $\Delta$ dotA/ $\Delta$ ravZ). These results illustrate that the co-infection experiment can be used to identify a L. pneumophila effector responsible for the restriction of LC3 recruitment to bacteria-containing vacuoles. These results also demonstrate that the restriction of LC3 recruitment to SCVs in the experimental condition can be explained solely by the function of the L. pneumophila effector RavZ, and any other effectors play only a limited role.

\section{Legionella Co-infection Restricts p62, NDP52, and Ubiquitin Recruitment to SCVs} Adaptor proteins such as p62 and NDP52 carry both LC3and ubiquitin-binding domains and are proposed to play a critical role in the recognition of invading microorganisms and 
A

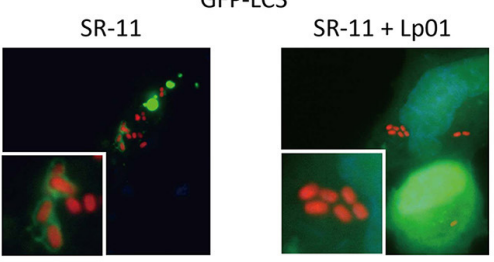

SR-11 + Lp01 $\Delta$ dotA

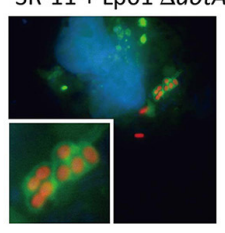

SR-11 + Lp01 $\Delta \operatorname{ravZ}$

B

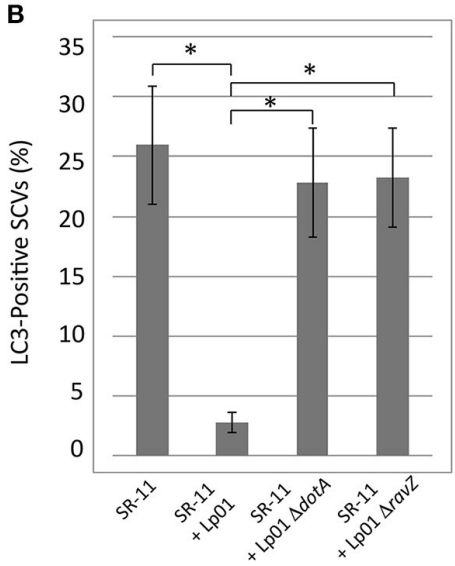

FIGURE 1 | Legionella interferes with LC3 recruitment to SCVs in a T4SS- and RavZ-dependent manner. HeLa cells expressing GFP-LC3 were co-infected with Salmonella expressing mCherry and Legionella for $1 \mathrm{~h}$. (A) LC3 recruitment to SCVs was visualized by fluorescence microscopy. (B) Average percentage of LC3-positive SCVs in the cells was calculated from three independent examinations. Data present the average $\pm S D$; ${ }^{*} P<0.02$

the induction of selective autophagy. We thus examined the effect of co-infecting L. pneumophila on the recruitment of these adaptor proteins to SCVs. A fraction of SCVs acquired GFP-p62 or GFP-NDP52 in HeLa cells producing GFP-p62 or GFP-NDP52 respectively at $1 \mathrm{~h}$ post-infection (Figure 2 SR-11). Intriguingly, the recruitment to SCVs of both GFPp62 and GFP-NDP52 was severely restricted by co-infecting L. pneumophila, as in the case of LC3 (Figures 2A,B,C SR$11+\mathrm{Lp} 01)$. The restriction was dependent on the presence of functional Dot/Icm T4SS in the co-infecting L. pneumophila (Figures 2A,B,C SR-11+Lp01 $\Delta \operatorname{dot} A$ ). RavZ disruption restored the GFP-p62 and GFP-NDP52 recruitment to extents similar to or somewhat less than those observed in HeLa cells infected solely with $S$. Typhimurium (Figures $\mathbf{2 A}, \mathbf{B}, \mathbf{C}$ SR-11+ $\Delta$ ravZ). We also examined the recruitment to SCVs of Tecpr1-GFP, another autophagy adaptor protein that does not possess ubiquitin-binding activity. In contrast to GFP-p62 and GFPNDP52, Tecpr1-GFP recruitment to SCVs was not affected by co-infecting L. pneumophila (Figures 2A,D). These results prompted us to examine ubiquitin recruitment to SCVs, an upstream event before adaptor recruitment, in the coinfection system. Using immunofluorescence microscopy with anti-conjugated-ubiquitin antibody (FK2), we found that the fraction of ubiquitin-positive SCVs was significantly reduced in the presence of co-infecting L. pneumophila, (Figures 3A,B SR-11+Lp01). As in the case of LC3, p62, and NDP52, the restriction required a functional Dot/Icm T4SS and the effector RavZ of co-infecting L. pneumophila (Figures 3A,B SR-11+Lp01 $\Delta \operatorname{dot} A / \Delta \operatorname{rav} Z)$.

\section{RavZ Restricts Ubiquitin Recruitment to SCVs}

The L. pneumophila effector RavZ appears to be necessary for the reduction of ubiquitin-positive SCVs in the coinfection condition. To test whether RavZ was sufficient for this reduction, we examined ubiquitin recruitment to SCVs in HeLa cells ectopically expressing RavZ (Figure 4A). The fraction of ubiquitin-positive SCVs in 3xFLAG-RavZ producing HeLa cells was lower $(\sim 5 \%)$ than that in vector-transfected cells $(\sim 30 \%)$, indicating that $\mathrm{RavZ}$ is sufficient for the restriction. RavZ deconjugates LC3, and the $\operatorname{Rav}_{\mathrm{C} 258 \mathrm{~A}}$ mutation disrupts this enzymatic activity. Ectopic expression of $3 x$ FLAG-RavZ $Z_{C 258 \mathrm{~A}}$ did not affect ubiquitin recruitment to SCVs, demonstrating that the same enzymatic activity is required for the restriction of ubiquitin recruitment to SCVs (Figure 4A).

To further characterize the role of RavZ in decreasing ubiquitin-positive SCVs, we used semi-permeabilized Salmonella-infected HeLa cells. The semi-permeabilized cells were washed to remove cytoplasmic proteins and compounds, and purified His-RavZ or His-RavZ $Z_{C 258 \mathrm{~A}}$ was added back at various concentrations. LC3 and ubiquitin levels on SCVs were determined by immunofluorescence staining using specific antibodies. Treatment with His-RavZ at a saturating concentration of $25 \mu \mathrm{g} / \mathrm{ml}(430 \mu \mathrm{M})$ decreased LC3 detectable on SCVs (Figure 4B, Figure S1A). In the same condition, the fraction of ubiquitin-positive SCVs was reduced twofold (Figure 4C). The added His-RavZ significantly reduced ubiquitin and LC3 levels on SCVs at a concentration as low as $0.008 \mu \mathrm{g} / \mathrm{ml}(140 \mathrm{nM})$, and the reactions were readily saturated as His-RavZ concentration increased (Figure S1), indicating the RavZ activity toward ubiquitin had an effective concentration comparable to that of its activity toward LC3. There was a residual fraction of ubiquitin-positive SCVs even at the saturated concentration of RavZ, suggesting that a certain fraction or species of ubiquitin cannot be removed by the action of RavZ (Figure S1B). Collectively, these results demonstrated that the activity of RavZ is necessary and sufficient for the reduction of ubiquitin levels on SCVs, presumably by enzymatically removing ubiquitin from unknown substrates on Salmonella or SCVs.

\section{RavZ Does Not Affect Ubiquitin Recruitment to LCVs}

We examined whether RavZ inhibits ubiquitin recruitment to LCVs in L. pneumophila-infected cells. In HeLa cells 


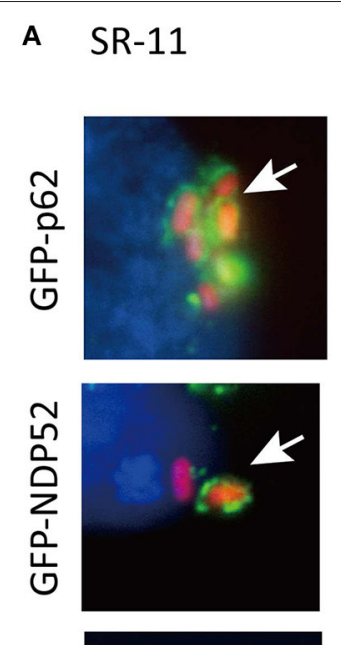

\section{SR-11}

$+\mathrm{LP} 01$
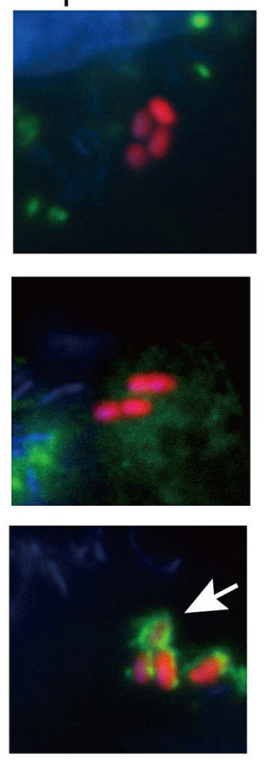

C

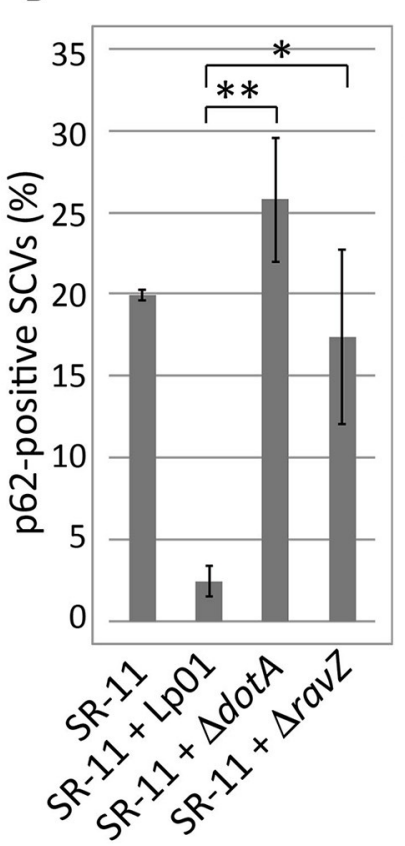

SR-11
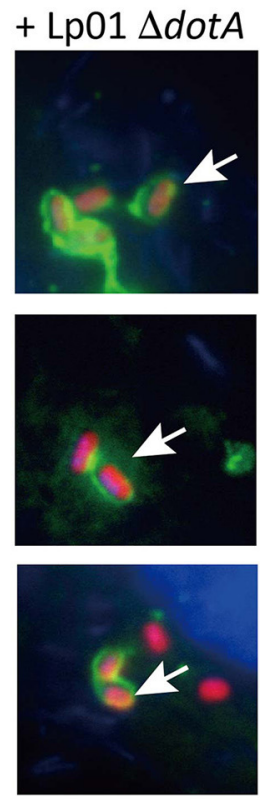

D
SR-11
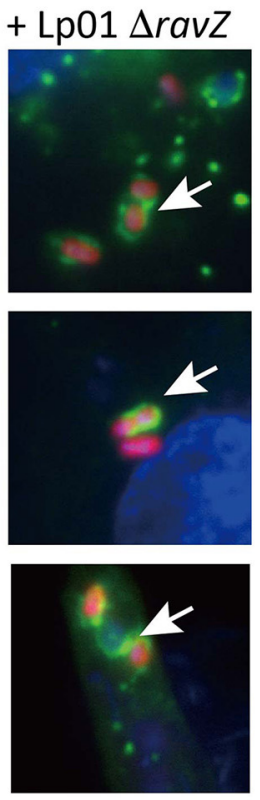
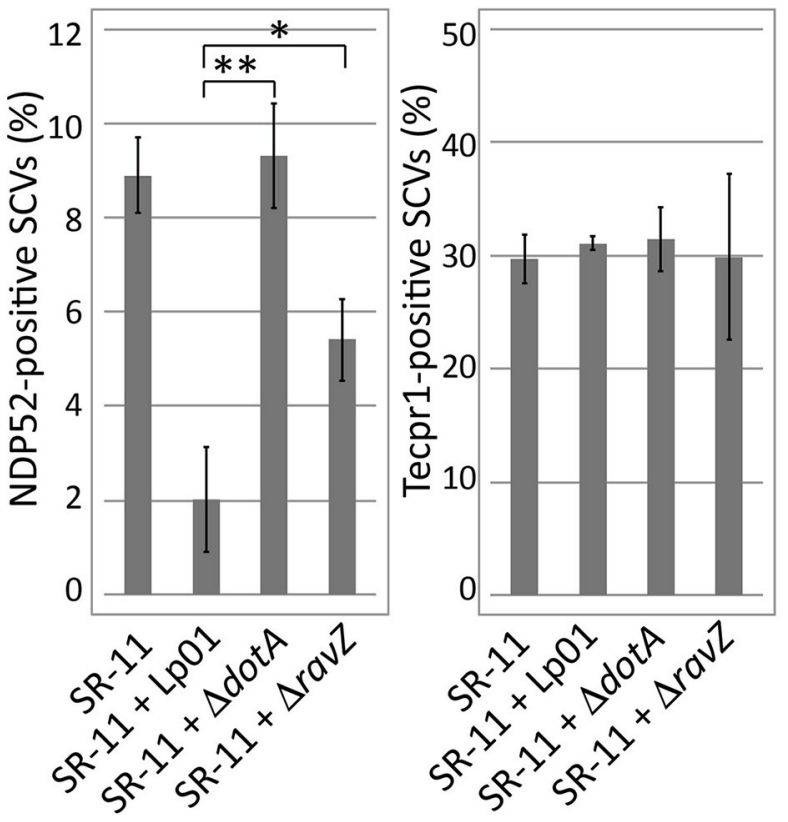

FIGURE 2 | Legionella interferes with the recruitment of ubiquitin-dependent adaptor proteins to SCVs in a T4SS- and RavZ-dependent manner. HeLa cells expressing GFP-p62, GFP-NDP52, or Tecpr1-GFP were co-infected with Salmonella expressing mCherry and Legionella for $1 \mathrm{~h}$. (A) Recruitment of the adaptor proteins to SCVs was visualized by fluorescence microscopy. Adaptor-positive SCVs are indicated by arrows. Average percentage of p62 (B), NDP52 (C), or Tecpr1 (D)-positive SCVs in transfected cells was calculated from three independent examinations. Data present the average $\pm S D ;{ }^{*} P<0.02,{ }^{* *} P<0.01$.

infected with a L. pneumophila $\triangle \operatorname{ravZ}$ strain, the frequency of ubiquitin-positive LCVs was not significantly affected by ectopically expressed 3xFLAG-RavZ or its catalytic mutant (Figure 4D). In mouse bone marrow-derived macrophages (BMDMs), infection with L. pneumophila resulted in the appearance of LCVs highly decorated with ubiquitin in a T4SSdependent manner (Figure 5). However, up to $10 \mathrm{~h}$ postinfection, there was no significant difference in the fractions of ubiquitin-positive LCVs containing wild-type and $\triangle \operatorname{rav} Z$ strains. It has been shown that ubiquitin recruitment to LCVs 


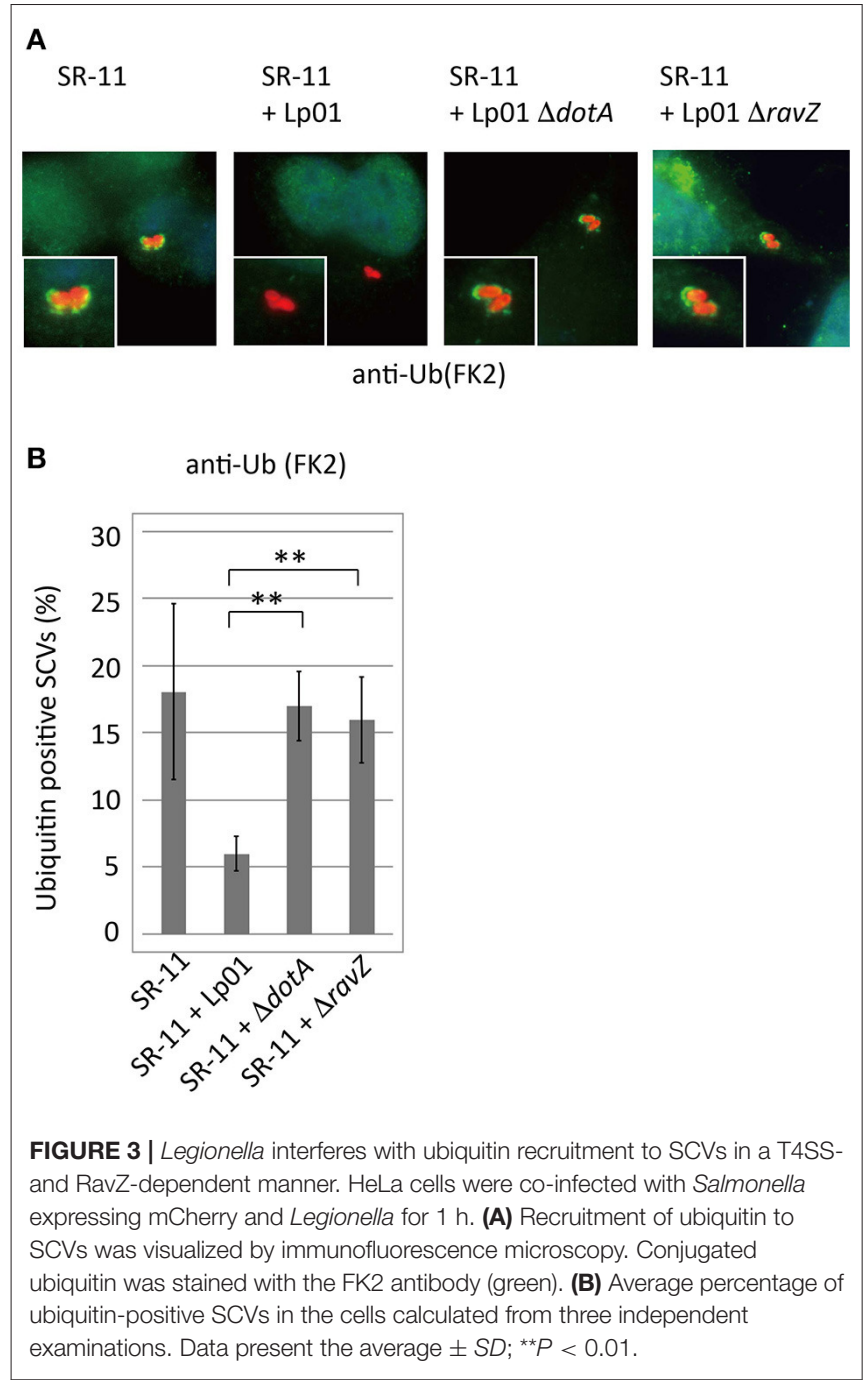

requires bacterial protein synthesis (Dorer et al., 2006). To examine the possibility that the persistent ubiquitin recruitment to LCVs obscures RavZ-dependent ubiquitin reduction, we repeated this experiment in the presence of chloramphenicol, a bacterial translation inhibitor, in order to restrict de novo supply of ubiquitin to LCVs (Figure S2). The fraction of ubiquitin-positive LCVs was strongly reduced in the presence of chloramphenicol, while again there was no significant difference in the fractions of ubiquitin-positive LCVs containing wildtype and $\triangle \operatorname{rav} Z$ strains. These results do not support a potential contribution of RavZ to removing ubiquitin from LCVs.

\section{The RavZ-Dependent Reduction of Ubiquitin-Positive SCVs Does Not Require a Functional LC3 Conjugation System}

Autophagy adaptor proteins p62 and NDP52 can bind both to ubiquitin and to LC3. RavZ possesses the enzymatic activity to irreversibly deconjugate LC3 from PE. Therefore, one of
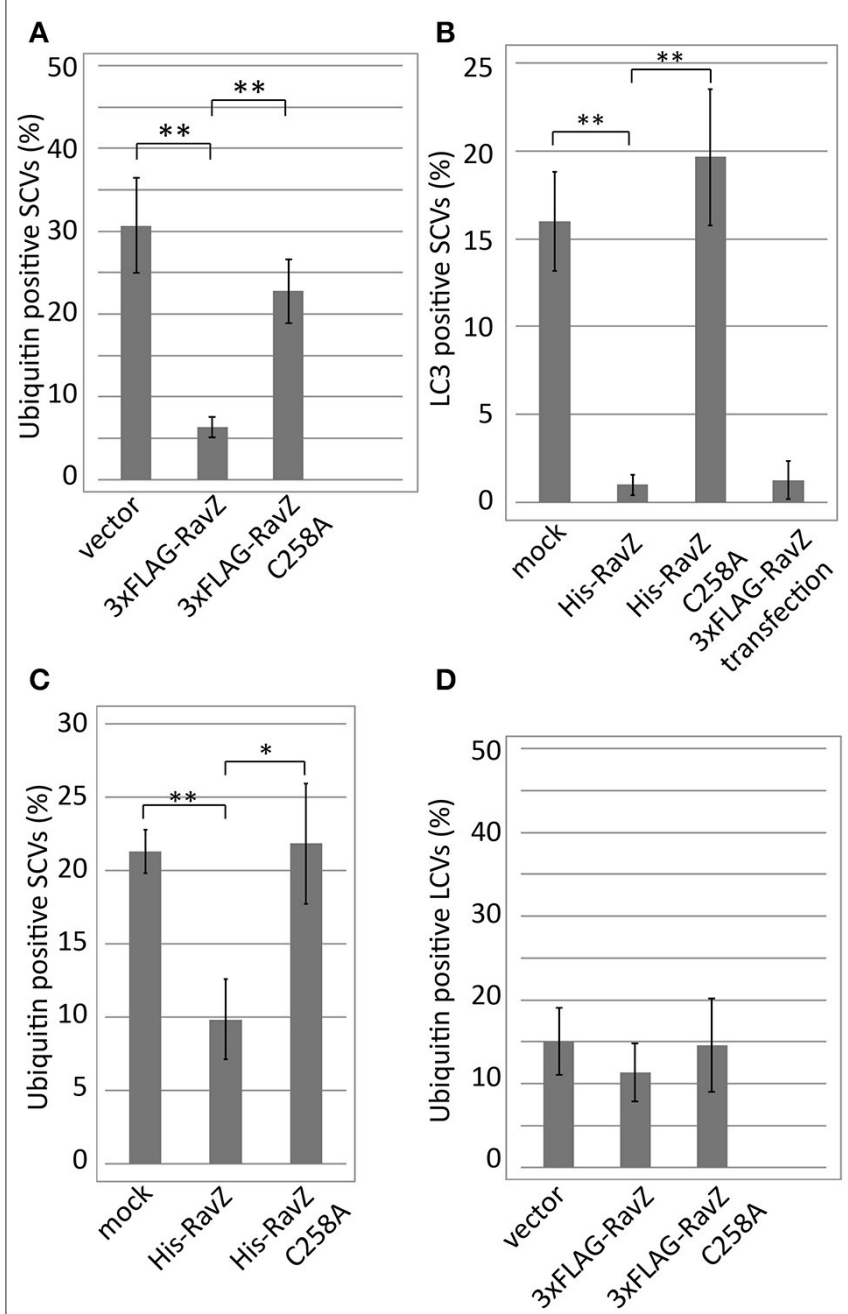

FIGURE 4 | Legionella RavZ appears to remove ubiquitin from SCVs in a manner dependent on the catalytic residue responsible for autophagy inhibition. (A,D) 3xFLAG-RavZ or its catalytic mutant was expressed in HeLa cells by transfection. The cells were infected with Salmonella expressing mCherry (A) or Legionella (D) for 1 h. (B,C) The purified His-tagged RavZ or its catalytic mutant protein was supplied into HeLa cells expressing GFP-LC3 (B) or HeLa cells (C) semi-permeabilized with Digitonin-containing buffer after $1 \mathrm{~h}$ of infection with Salmonella expressing mCherry. Conjugated ubiquitin was stained with the FK2 antibody. Average percentage of LC3 (B) or ubiquitin (A,C,D)-positive SCVs or LCVs calculated from three independent examinations. Data present the average $\pm S D$; ${ }^{\star} P<0.02,{ }^{\star \star} P<0.01$.

the possible explanations for the reduction of ubiquitin-positive SCVs in the presence of functional RavZ is that RavZ-dependent deconjugation of LC3 leads to destabilization or dissociation of ubiquitinated complexes containing adaptor proteins in the vicinity of SCVs. Alternatively, the apparent decrease in ubiquitin could be attributed to loss of ubiquitinated LC3 from SCVs. These feedback scenarios predict that the reduction of LC3positive SCVs is a prerequisite for the reduction of ubiquitinpositive $\mathrm{SCVs}$. To test this possibility, we examined the ubiquitin levels on SCVs in Atg7 knockout (KO) MEF cells, in which LC3 conjugation to PE does not take place (Komatsu et al., 


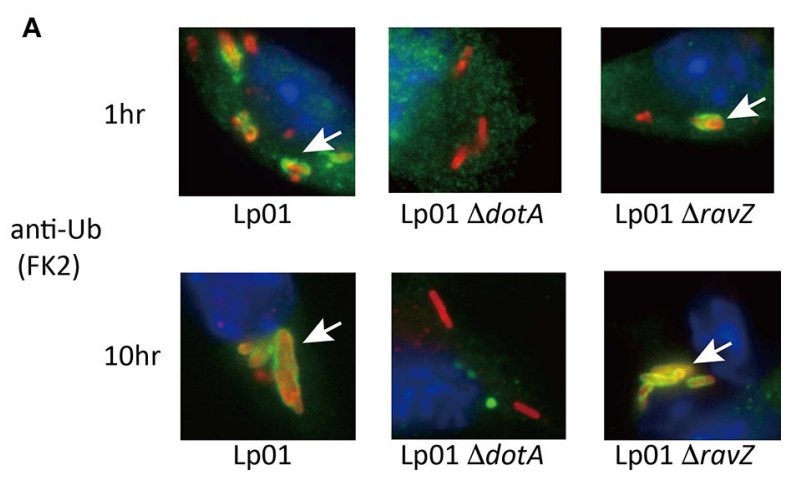

B

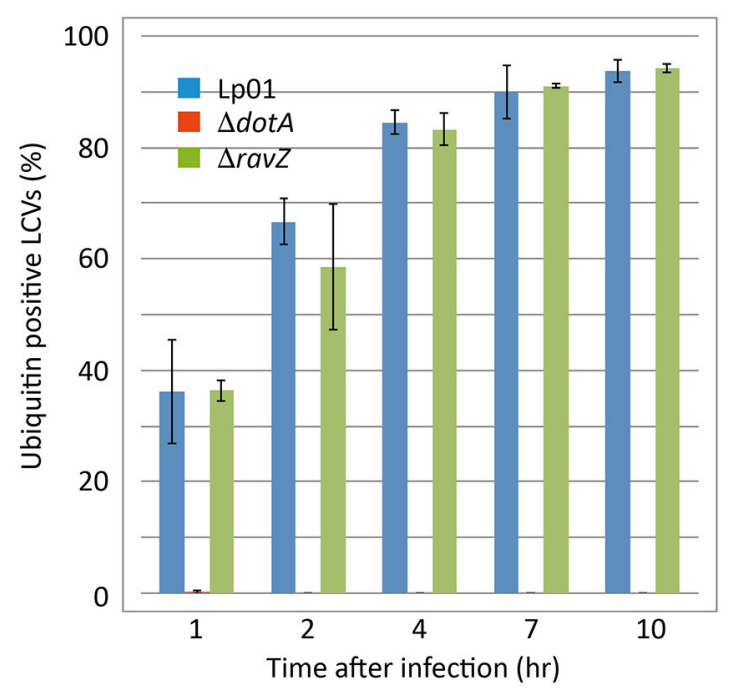

FIGURE 5 | RavZ appears not to affect ubiquitin recruitment to LCVs. Mouse bone marrow-derived macrophages were infected with Legionella for the indicated hours. (A) Recruitment of ubiquitin to LCVs was visualized by immunofluorescence microscopy. Legionella was stained with anti-Legionella antibody (red). Conjugated ubiquitin was stained with the FK2 antibody (green). Ubiquitin-positive LCVs are indicated by arrows. (B) Percentage of ubiquitin-positive LCVs. Data present the average $\pm S D$ calculated from three independent experiments.

2005) (Figure 6). We found that the RavZ-dependent reduction of ubiquitin-positive SCVs was observed both in wild-type and Atg7 KO MEF cells (Figure 6). This strongly suggests that the RavZ-dependent restriction of ubiquitin-positive SCVs does not result from the RavZ-mediated deconjugation of LC3 from PE.

\section{DISCUSSION}

Autophagy is carried out by a set of Atg proteins. Among the $\sim 30$ Atg proteins, the ubiquitin-like proteins Atg12 and Atg8 have important roles in the process (Geng and Klionsky, 2008). Similarly to the proteins involved in ubiquitin conjugation reactions, E1 and E2-like Atg proteins (Atg7 and Atg3/10 respectively) have been identified (Shintani et al., 1999; Tanida
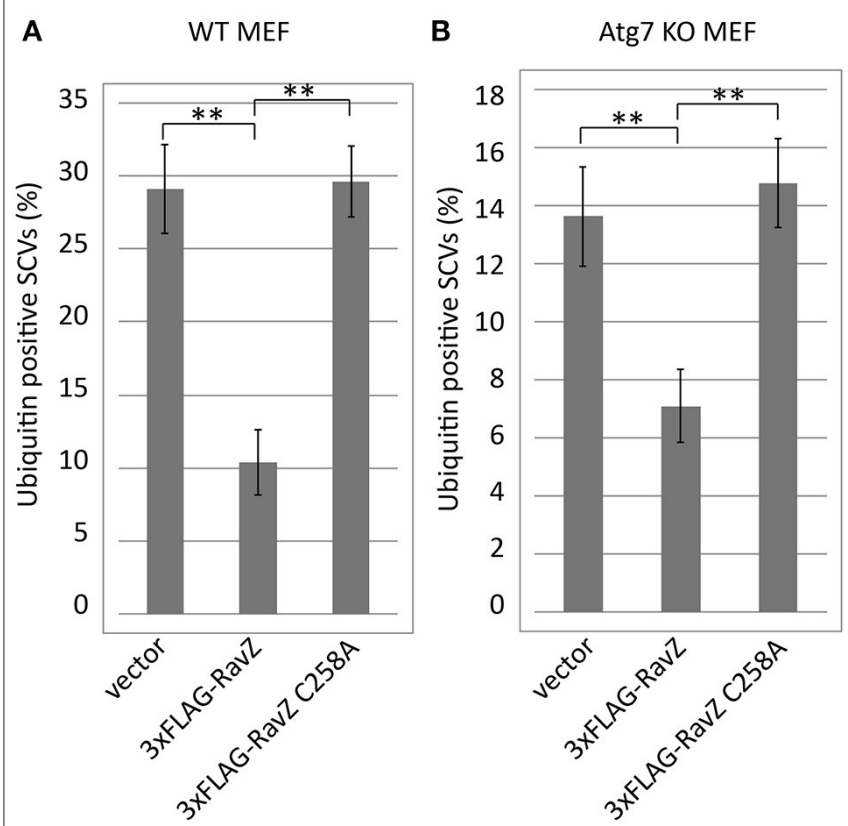

FIGURE 6 | Deficiency for LC3 maturation is not a cause of RavZ-mediated ubiquitin reduction on SCVs. Wild-type (A) or Atg7 knockout (B) MEF cells expressing RavZ or its catalytic mutant were infected with Salmonella expressing mCherry for $1 \mathrm{~h}$. Conjugated ubiquitin was stained with the FK2 antibody. Percentage of ubiquitin-positive SCVs calculated in three independent experiments. Data present the average $\pm S D$; ${ }^{\star \star} P<0.01$.

et al., 1999; Ichimura et al., 2000; Geng and Klionsky, 2008). Atg8 is a yeast counterpart of LC3, and structural studies of Atg8 family proteins including LC3 (Kouno et al., 2005) revealed structural similarity to ubiquitin (Shpilka et al., 2011). Atg8-PE conjugation is a crucial step for autophagosome maturation and is a reversible by the deconjugation enzyme Atg4 (Nakatogawa et al., 2007). RavZ was found to possess Atg4-like activity to deconjugate Atg8 family proteins from PE. There were several critical differences in their enzymatic activities. RavZ cleaves a distinct amide bond of LC3-PE from that targeted by Atg4 (Choy et al., 2012). Therefore, LC3 deconjugation by RavZ is an irreversible process in which deconjugated LC3 is no longer available for reconjugation to PE. Furthermore, RavZ cleaves LC3 conjugated to PE but not to another protein such as YFP, whereas Atg4 can cleave both LC3-PE and LC3-YFP.

The experiment using semi-permeabilized cells demonstrated that pre-existing ubiquitin on SCVs can be targeted by RavZ and that the reduction in ubiquitin-positive SCVs is dependent on the active site of RavZ, which is known to be responsible for LC3 delipidation (Figure 4). Furthermore, the effect was observed in the absence of LC3 conjugation to SCVs (Figure 6). One possible explanation for these results is that the known amide-bond-cleavage activity of RavZ targets not only LC3-PE but also ubiquitin-conjugated molecules on SCVs. The recently solved crystal structure of RavZ suggests mechanisms for how RavZ preferentially targets the early autophagosomal membrane to cleave lipid-conjugated LC3 (Horenkamp et al., 2015). The structure contained a catalytic domain that showed structural 
similarity with Ulp family deubiquitinase-like enzymes and a Cterminal domain including a phosphatidylinositol 3-phosphate (PI3P) binding site. These and our results raise the possibility that RavZ has deubiquitinase-like enzymatic activity depending on binding to PI3P present on bacterial vacuoles. A recent report about chemical approaches using semisynthetic LC3 proteins with various modifications described that RavZ activity is strictly dependent on the lipid structure of the substrate (Yang et al., 2017). Together with the fact that RavZ recognizes the LC3 conjugated to a phosphoglycerolipid PE but not LC3-protein conjugates (Choy et al., 2012), it is tempting to speculate that RavZ may have activity toward ubiquitin conjugated to some phosphoglycerolipids containing an amine group, for example PE and phosphatidylserine (PS) in bacteria-containing vacuoles. Cellular polyubiquitin levels are not affected by ectopic expression of RavZ, indicating that RavZ is not likely to be a canonical deubiquitinase (Figure S3). Such a ubiquitin-lipid conjugate might play a role as a signal for selective autophagy. Although this is an intriguing hypothesis, the possibility still remains that RavZ might play an indirect role in ubiquitin removal from SCVs, in a manner dependent on the same active site for LC3 deconjugation but not on the removal of LC3. It was reported that a subset of cytosolic Salmonella associates with autophagy proteins ( $\mathrm{Yu}$ et al., 2014). In this context, ubiquitin or LC3 located on not only bacterial vacuoles but also cytosolic bacteria might be targeted by RavZ activity. Future studies to clarify the molecular mechanisms of ubiquitin removal from bacterial-containing vacuoles mediated by RavZ may shed light on the recognition process of selective autophagy, an important host response for restricting invading microorganisms.

\section{AUTHOR CONTRIBUTIONS}

TK and HN designed research; TK, XB, AH, and HN performed research; TK, $\mathrm{XB}$, and $\mathrm{HN}$ analyzed data; and TK and $\mathrm{HN}$ wrote the paper.

\section{REFERENCES}

Berger, K. H., and Isberg, R. R. (1993). Two distinct defects in intracellular growth complemented by a single genetic locus in Legionella pneumophila. Mol. Microbiol. 7, 7-19. doi: 10.1111/j.1365-2958.1993.tb01092.x

Birmingham, C. L., Smith, A. C., Bakowski, M. A., Yoshimori, T., and Brumell, J. H. (2006). Autophagy controls Salmonella infection in response to damage to the Salmonella-containing vacuole. J. Biol. Chem. 281, 11374-11383. doi: 10.1074/jbc.M509157200

Cemma, M., Kim, P. K., and Brumell, J. H. (2011). The ubiquitin-binding adaptor proteins p62/SQSTM1 and NDP52 are recruited independently to bacteriaassociated microdomains to target Salmonella to the autophagy pathway. Autophagy 7, 341-345. doi: 10.4161/auto.7.3.14046

Choy, A., Dancourt, J., Mugo, B., O’Connor, T. J., Isberg, R. R., Melia, T. J., et al. (2012). The Legionella effector RavZ inhibits host autophagy through irreversible Atg8 deconjugation. Science 338, 1072-1076. doi: $10.1126 /$ science. 1227026

Deretic, V. (2010). Autophagy in infection. Curr. Opin. Cell Biol. 22, 252-262. doi: 10.1016/j.ceb.2009.12.009

Dolinsky, S., Haneburger, I., Cichy, A., Hannemann, M., Itzen, A., and Hilbi, H. (2014). The Legionella longbeachae Icm/Dot substrate SidC selectively binds phosphatidylinositol 4-phosphate with nanomolar affinity and promotes

\section{FUNDING}

This work was supported by MEXT/JSPS KAKENHI Grant numbers 26670212, 15H04728, 23117002 (to HN), 15H01322, 25460530, 16H05189, and 16K14724 (to TK).

\section{ACKNOWLEDGMENTS}

We thank Michinaga Ogawa for providing GFP-LC3, GFPp62, GFP-NDP52, and Tecpr1-GFP expression plasmids. Immortalized Atg7-deficient MEFs were kindly provided by Tatsuya Saitoh on behalf of Masaaki Komatsu. We thank Craig Roy, Hiroki Ando, and Tomoe Kitao for critical discussions, Akiko Okura and Masayo Yamada for technical assistance.

\section{SUPPLEMENTARY MATERIAL}

The Supplementary Material for this article can be found online at: http://journal.frontiersin.org/article/10.3389/fcimb. 2017.00384/full\#supplementary-material

Figure S1 | Concentration-dependency of the effect of RavZ treatment of semi-permeabilized cells on the level of ubiquitin-positive SCVs. After $1 \mathrm{~h}$ of infection with Salmonella expressing mCherry, HeLa cells expressing GFP-LC3 were semi-permeabilized with Digitonin-containing buffer and treated with purified His-RavZ at the indicated concentrations. Conjugated ubiquitin was stained with the FK2 antibody. Percentage of LC3 (A) or ubiquitin (B)-positive SCVs calculated from three independent experiments. Data present the average $\pm \mathrm{SD}$.

Figure S2 | RavZ appears not to affect ubiquitin recruitment to LCVs even in the absence of de novo supply of ubiquitin on LCVs. Mouse bone marrow-derived macrophages were infected with Legionella for 7 hs in the absence (dark gray) or presence of $25 \mu \mathrm{g} / \mathrm{ml}$ of chloramphenicol (light gray). Conjugated ubiquitin was stained with the FK2 antibody. Average percentage of ubiquitin-positive LCVs was calculated from three independent examinations. Data present the average $\pm S D$.

Figure S3 | Cellular polyubiquitin levels are not affected by RavZ. HEK293-Fc $\gamma$ RII cells were transfected with p3xFLAG-CMV-10 expression vector or derived 3xFLAG-RavZ or RavZ C258A expressing plasmids for $24 \mathrm{~h}$. Cell lysates were applied on 10-20\% gradient polyacrylamide gel electrophoresis followed by immunoblotting using anti-ubiquitin antibody (P4D1) for detection of mono and polyubiquitins and anti- $\alpha$-tubulin for internal loading control.

pathogen vacuole-endoplasmic reticulum interactions. Infect. Immun. 82, 4021-4033. doi: 10.1128/IAI.01685-14

Dorer, M. S., Kirton, D., Bader, J. S., and Isberg, R. R. (2006). RNA interference analysis of Legionella in Drosophila cells: exploitation of early secretory apparatus dynamics. PLoS Pathog. 2:e34. doi: 10.1371/journal.ppat.00 20034

Ensminger, A. W., and Isberg, R. R. (2009). Legionella pneumophila Dot/Icm translocated substrates: a sum of parts. Curr. Opin. Microbiol. 12, 67-73. doi: 10.1016/j.mib.2008.12.004

Geng, J., and Klionsky, D. J. (2008). The Atg8 and Atg12 ubiquitin-like conjugation systems in macroautophagy. 'Protein modifications: beyond the usual suspects' review series. EMBO Rep. 9, 859-864. doi: 10.1038/embor.2008.163

He, C., and Klionsky, D. J. (2009). Regulation mechanisms and signaling pathways of autophagy. Annu. Rev. Genet. 43, 67-93. doi: 10.1146/annurev-genet-102808-114910

Horenkamp, F. A., Kauffman, K. J., Kohler, L. J., Sherwood, R. K., Krueger, K. P., Shteyn, V., et al. (2015). The Legionella anti-autophagy effector RavZ targets the autophagosome via PI3P- and curvature-sensing motifs. Dev. Cell 34, 569-576. doi: 10.1016/j.devcel.2015.08.010

Horenkamp, F. A., Mukherjee, S., Alix, E., Schauder, C. M., Hubber, A. M., Roy, C. R., et al. (2014). Legionella pneumophila subversion of host vesicular transport by SidC effector proteins. Traffic 15, 488-499. doi: 10.1111/tra.12158 
Horwitz, M. A., and Silverstein, S. C. (1983). Intracellular multiplication of Legionnaires' disease bacteria (Legionella pneumophila) in human monocytes is reversibly inhibited by erythromycin and rifampin. J. Clin. Invest. 71, 15-26. doi: $10.1172 / J C I 110744$

Hsu, F., Luo, X., Qiu, J., Teng, Y. B., Jin, J., Smolka, M. B., et al. (2014). The Legionella effector SidC defines a unique family of ubiquitin ligases important for bacterial phagosomal remodeling. Proc. Natl. Acad. Sci. U.S.A. 111, 10538-10543. doi: 10.1073/pnas. 1402605111

Huang, J., and Brumell, J. H. (2014). Bacteria-autophagy interplay: a battle for survival. Nat. Rev. Microbiol. 12, 101-114. doi: 10.1038/nrmicro3160

Hubber, A., Arasaki, K., Nakatsu, F., Hardiman, C., Lambright, D., De Camilli, P., et al. (2014). The machinery at endoplasmic reticulum-plasma membrane contact sites contributes to spatial regulation of multiple Legionella effector proteins. PLoS Pathog. 10:e1004222. doi: 10.1371/journal.ppat.1004222

Hubber, A., Kubori, T., Coban, C., Matsuzawa, T., Ogawa, M., Kawabata, T., et al. (2017). Bacterial secretion system skews the fate of Legionellacontaining vacuoles towards LC3-associated phagocytosis. Sci. Rep. 7:44795. doi: $10.1038 /$ srep 44795

Hubber, A., Kubori, T., and Nagai, H. (2013). Modulation of the ubiquitination machinery by Legionella. Curr. Top. Microbiol. Immunol. 376, 227-247. doi: $10.1007 / 82 \_2013 \_343$

Hubber, A., and Roy, C. R. (2010). Modulation of host cell function by Legionella pneumophila type IV effectors. Annu. Rev. Cell Dev. Biol. 26, 261-283. doi: 10.1146/annurev-cellbio-100109-104034

Ichimura, Y., Kirisako, T., Takao, T., Satomi, Y., Shimonishi, Y., Ishihara, N., et al. (2000). A ubiquitin-like system mediates protein lipidation. Nature 408, 488-492. doi: 10.1038/35044114

Isberg, R. R., O'Connor, T. J., and Heidtman, M. (2009). The Legionella pneumophila replication vacuole: making a cosy niche inside host cells. Nat. Rev. Microbiol. 7, 13-24. doi: 10.1038/nrmicro1967

Kabeya, Y., Mizushima, N., Ueno, T., Yamamoto, A., Kirisako, T., Noda, T., et al. (2000). LC3, a mammalian homologue of yeast Apg8p, is localized in autophagosome membranes after processing. EMBO J. 19, 5720-5728. doi: 10.1093/emboj/19.21.5720

Komatsu, M., Waguri, S., Ueno, T., Iwata, J., Murata, S., Tanida, I., et al. (2005). Impairment of starvation-induced and constitutive autophagy in Atg7deficient mice. J. Cell Biol. 169, 425-434. doi: 10.1083/jcb.200412022

Kotewicz, K. M., Ramabhadran, V., Sjoblom, N., Vogel, J. P., Haenssler, E., Zhang, M., et al. (2017). A single legionella effector catalyzes a multistep ubiquitination pathway to rearrange tubular endoplasmic reticulum for replication. Cell Host Microbe 21, 169-181. doi: 10.1016/j.chom.2016.12.007

Kouno, T., Mizuguchi, M., Tanida, I., Ueno, T., Kanematsu, T., Mori, Y., et al. (2005). Solution structure of microtubule-associated protein light chain 3 and identification of its functional subdomains. J. Biol. Chem. 280, 24610-24617. doi: 10.1074/jbc.M413565200

Kuballa, P., Nolte, W. M., Castoreno, A. B., and Xavier, R. J. (2012). Autophagy and the immune system. Annu. Rev. Immunol. 30, 611-646. doi: 10.1146/annurev-immunol-020711-074948

Kubori, T., Hyakutake, A., and Nagai, H. (2008). Legionella translocates an E3 ubiquitin ligase that has multiple U-boxes with distinct functions. Mol. Microbiol. 67, 1307-1319. doi: 10.1111/j.1365-2958.2008.06124.x

Luo, X., Wasilko, D. J., Liu, Y., Sun, J., Wu, X., Luo, Z. Q., et al. (2015). Structure of the legionella virulence factor, sidc reveals a unique PI(4)P-specific binding domain essential for its targeting to the bacterial phagosome. PLoS Pathog. 11:e1004965. doi: $10.1145 / 2818302$

Mesquita, F. S., Thomas, M., Sachse, M., Santos, A. J., Figueira, R., and Holden, D. W. (2012). The Salmonella deubiquitinase SseL inhibits selective autophagy of cytosolic aggregates. PLoS Pathog. 8:e1002743. doi: 10.1371/journal.ppat.1002743

Nakatogawa, H., Ichimura, Y., and Ohsumi, Y. (2007). Atg8, a ubiquitin-like protein required for autophagosome formation, mediates membrane tethering and hemifusion. Cell 130, 165-178. doi: 10.1016/j.cell.2007.05.021

Narayanan, L. A., and Edelmann, M. J. (2014). Ubiquitination as an efficient molecular strategy employed in Salmonella infection. Front. Immunol. 5:558. doi: 10.3389/fimmu.2014.00558

Ogawa, M., Yoshikawa, Y., Kobayashi, T., Mimuro, H., Fukumatsu, M., Kiga, K., et al. (2011). A Tecpr1-dependent selective autophagy pathway targets bacterial pathogens. Cell Host Microbe 9, 376-389. doi: 10.1016/j.chom.2011.0 4.010

Price, C. T., Al-Quadan, T., Santic, M., Rosenshine, I., and Abu Kwaik, Y. (2011). Host proteasomal degradation generates amino acids essential for intracellular bacterial growth. Science 334, 1553-1557. doi: 10.1126/science.1212868

Qiu, J., Sheedlo, M. J., Yu, K., Tan, Y., Nakayasu, E. S., Das, C., et al. (2016). Ubiquitination independent of E1 and E2 enzymes by bacterial effectors. Nature 533, 120-124. doi: 10.1038/nature17657

Ragaz, C., Pietsch, H., Urwyler, S., Tiaden, A., Weber, S. S., and Hilbi, H. (2008). The Legionella pneumophila phosphatidylinositol-4 phosphatebinding type IV substrate SidC recruits endoplasmic reticulum vesicles to a replication-permissive vacuole. Cell. Microbiol. 10, 2416-2433. doi: 10.1111/j.1462-5822.2008.01219.x

Rolando, M., Escoll, P., Nora, T., Botti, J., Boitez, V., Bedia, C., et al. (2016). Legionella pneumophila S1P-lyase targets host sphingolipid metabolism and restrains autophagy. Proc. Natl. Acad. Sci. U.S.A. 113, 1901-1906. doi: $10.1073 /$ pnas.1522067113

Sheedlo, M. J., Qiu, J., Tan, Y., Paul, L. N., Luo, Z. Q., and Das, C. (2015). Structural basis of substrate recognition by a bacterial deubiquitinase important for dynamics of phagosome ubiquitination. Proc. Natl. Acad. Sci. U.S.A. 112, 15090-15095. doi: 10.1073/pnas.1514568112

Shintani, T., Mizushima, N., Ogawa, Y., Matsuura, A., Noda, T., and Ohsumi, Y. (1999). Apg10p, a novel protein-conjugating enzyme essential for autophagy in yeast. $E M B O ~ J .18,5234-5241$. doi: $10.1093 / \mathrm{emboj} / 18.19 .5234$

Shpilka, T., Weidberg, H., Pietrokovski, S., and Elazar, Z. (2011). Atg8: an autophagy-related ubiquitin-like protein family. Gen. Biol. 12, 226. doi: $10.1186 /$ gb-2011-12-7-226

Tanida, I., Mizushima, N., Kiyooka, M., Ohsumi, M., Ueno, T., Ohsumi, Y., et al. (1999). Apg7p/Cvt2p: a novel protein-activating enzyme essential for autophagy. Mol. Biol. Cell 10, 1367-1379. doi: 10.1091/mbc.10.5.1367

Thurston, T. L., Ryzhakov, G., Bloor, S., von Muhlinen, N., and Randow, F. (2009). The TBK1 adaptor and autophagy receptor NDP52 restricts the proliferation of ubiquitin-coated bacteria. Nat. Immunol. 10, 1215-1221. doi: 10.1038/ni.1800

Thurston, T. L., Wandel, M. P., von Muhlinen, N., Foeglein, A., and Randow, F. (2012). Galectin 8 targets damaged vesicles for autophagy to defend cells against bacterial invasion. Nature 482, 414-418. doi: 10.1038/nature 10744

Weber, S. S., Ragaz, C., Reus, K., Nyfeler, Y., and Hilbi, H. (2006). Legionella pneumophila exploits $\mathrm{PI}(4) \mathrm{P}$ to anchor secreted effector proteins to the replicative vacuole. PLoS Pathog. 2:e46. doi: 10.1371/journal.ppat.0020046

Yang, A., Pantoom, S., and Wu, Y. W. (2017). Elucidation of the anti-autophagy mechanism of the Legionella effector RavZ using semisynthetic LC3 proteins. Elife 6:e23905. doi: 10.7554/eLife.23905

Yu, H. B., Croxen, M. A., Marchiando, A. M., Ferreira, R. B., Cadwell, K., Foster, L. J., et al. (2014). Autophagy facilitates Salmonella replication in HeLa cells. mBio 5, e00865-e00814. doi: 10.1128/mBio.00865-14

Zheng, Y. T., Shahnazari, S., Brech, A., Lamark, T., Johansen, T., and Brumell, J. H. (2009). The adaptor protein p62/SQSTM1 targets invading bacteria to the autophagy pathway. J. Immunol. 183, 5909-5916. doi: 10.4049/jimmunol.0900441

Zhou, Y., and Zhu, Y. (2015). Diversity of bacterial manipulation of the host ubiquitin pathways. Cell. Microbiol. 17, 26-34. doi: 10.1111/cmi.12384

Zuckman, D. M., Hung, J. B., and Roy, C. R. (1999). Pore-forming activity is not sufficient for Legionella pneumophila phagosome trafficking and intracellular growth. Mol. Microbiol. 32, 990-1001. doi: 10.1046/j.1365-2958.1999. 01410.x

Conflict of Interest Statement: The authors declare that the research was conducted in the absence of any commercial or financial relationships that could be construed as a potential conflict of interest.

Copyright (c) 2017 Kubori, Bui, Hubber and Nagai. This is an open-access article distributed under the terms of the Creative Commons Attribution License (CC BY). The use, distribution or reproduction in other forums is permitted, provided the original author(s) or licensor are credited and that the original publication in this journal is cited, in accordance with accepted academic practice. No use, distribution or reproduction is permitted which does not comply with these terms. 